\section{THU0447 CO-MORBID GOUT IS ASSOCIATED WITH INCREASED CARDIOVASCULAR RISK FACTORS IN PATIENTS WITH TYPE 2 DIABETES, BUT NOT CARDIOVASCULAR EVENTS OR MORTALITY}

M. Mian ${ }^{1}$, S.K. Patel ${ }^{2}$, B. Wai ${ }^{1}$, P.M. Srivastava ${ }^{1}$, R.R.C. Buchanan ${ }^{1,2}$, L.M. Burrell $1,2 .{ }^{1}$ Department of Medicine, Austin Health; ${ }^{2}$ Department of Medicine, University of Melbourne, Melbourne, Australia

Background: Gout is an inflammatory arthropathy characterised by elevated serum uric acid levels. In Australia, gout has a prevalence of $1.7-4 \%{ }^{1}$ This increased to $\sim 10 \%$ in community based Australian patients with type 2 diabetes, although elevated serum uric acid did not predict cardiovascular (CV) or all-cause mortality. ${ }^{2}$ To date, the long-term outcomes of patients with diabetes and comorbid gout being followed up in the hospital out-patient setting have not been studied. Objectives: To compare cardiovascular risk factors and long-term outcomes and mortality in patients with type 2 diabetes according to the presence or absence of gout.

Methods: 1,405 patients with type 2 diabetes were prospectively recruited from the outpatient setting at Austin Hospital. Baseline cardiovascular risk factors and comorbidities were identified. Patients were classified as having gout if they gave a history of gout or were taking medication for the treatment of gout. For statistical analysis, patients with diabetes (Group 1) were compared to those with diabetes and gout (Group 2). Cardiovascular events and long-term CV mortality were assessed over a 10 year period.

Results: There were 1,329 patients with diabetes (Group 1; 95\%) and 76 with diabetes and gout (Group 2; $5 \%)$. Patients with gout were older $(68 \pm 11$ vs. $64 \pm 12 y, p=0.004)$, more likely to be male ( $80 \%$ vs. $59 \%, p<0.0001)$, with higher triglyceride levels (2.6 vs $1.9 \mathrm{mmol} / \mathrm{L}, \mathrm{p}=0.002$ ), lower $\mathrm{HDL}$ ( $1.05 \mathrm{vs} .1 .24 \mathrm{mmol} / \mathrm{L}$, $p<0.0001$ ), higher BMI (33 vs. $31, p=0.026$ ), and were more likely to have nephropathy $(55 \%(n=35)$ vs. $26 \%(n=311), p<0.0001)$ with increased albumin creatinine ratio $(3.4$ vs $1.8 \mathrm{~g} / \mathrm{mmol}, \mathrm{p}=0.002)$. Despite the worse cardiovascular risk profile in those with gout and diabetes, cardiovascular events and all-cause mortality were not significantly different between the groups (Group 1, 27\% ( $n=333)$ vs. $35 \%(n=23)$ in Group $2, p=0.201)$.

Conclusions: Although patients with comorbid gout and type 2 diabetes have a worse cardiovascular risk factor profile compared to those with diabetes alone, this was not associated with increased cardiovascular morbidity or all-cause mortality. These results suggest that elevated uric acid and gout are markers rather than determinants of $\mathrm{CV}$ mortality.

\section{References:}

[1] Robinson PC, Taylor WJ, Merriman TR. Systemic review of the prevalence of gout and hyperuricaemia in Australia. Internal Med J 2012; 42(9):997-1007.

[2] Ong G, Davis WA, Davis TME. Serum uric acid does not predict cardiovascular or all-cause mortality in type 2 diabetes: the Fremantle Diabetes Study. Diabetologia 2010; 53: 1288-129.

Disclosure of Interest: None declared

DOI: 10.1136/annrheumdis-2017-eular.3596

\section{THU0448 CHARACTERIZATION OF PATIENTS WITH CHRONIC REFRACTORY GOUT WHO DO AND DO NOT HAVE CLINICALLY APPARENT TOPHI: RESPONSE TO PEGLOTICASE}

N.L. Edwards ${ }^{1}$, J. Singh ${ }^{2}$, O. Troum ${ }^{3}$, A. Yeo ${ }^{4}$, P. Lipsky ${ }^{5} \cdot{ }^{1}$ Department of Medicine, University of Florida, Gainesville, FL; ${ }^{2}$ University of Alabama at Birmingham, Birmingham, AL; ${ }^{3}$ Keck School of Medicine, University of Southern California, Los Angeles, CA; ${ }^{4}$ Horizon Pharma, Lake Forest, IL; ${ }^{5}$ AMPEL BioSolutions, LLC, Charlottesville, VA, United States

Background: The term "chronic refractory gout" defines a subset of chronic gout patients who are either intolerant of or unresponsive to standard uric acid (UA) lowering therapy (ULT). Subjects $(\mathrm{N}=85)$ meeting this definition were enrolled in a study of pegloticase ( $8 \mathrm{mg}$ every 2 weeks [q2w], the approved dose), a mammalian recombinant uricase conjugated to polyethylene glycol that is approved for the treatment of gout refractory to conventional oral ULT. Of this group of subjects, $73 \%$ had clinically apparent tophi, whereas $27 \%$ did not.

Objectives: To determine the clinical characteristics and response to pegloticase therapy in patients with chronic refractory gout with and without clinically apparent tophi.

Methods: This analysis used results from two pivotal randomized controlled trials ${ }^{1}$ to assess the clinical characteristics and the efficacy of pegloticase ( $8 \mathrm{mg} \mathrm{q2w})$ in patients with chronic refractory gout with or without tophi at baseline. The results for serum urate (UA), flares, Patient Global Assessment (PGA), tender and swollen joints (TJC and SJC), bodily pain, Health Assessment Questionnaire-Disability Index (HAQ-DI), and the Arthritis-Specific Health Index (ASHI) and Bodily Pain from the Medical Outcomes Study Short Form 36 item (SF-36) were determined for each group.

Results: The analysis included patients with chronic refractory gout, 62 with tophi at baseline and 23 without tophi. Chronic refractory gout patients in the two groups were similar at baseline, with the only significant differences in mean values between tophaceous and nontophaceous gout groups as follows: TJC, 14.2 vs 5.00 ( $P=0.01)$; SJC, 10.9 vs 3.4 ( $P=0.003)$; ASHI, 50.4 vs $64.7(P=0.03)$; and HAQ-DI, 1.3 vs 0.6 , respectively $(P=0.001)$. Other measures of disease impact and comorbidities were not significantly different between groups. Treatment with pegloticase $8 \mathrm{mg}$ q2w resulted in significant and comparable reductions in serum UA in both groups. Comparison of results from baseline and after 6 months of treatment for patients with tophi at baseline indicated significant reductions in serum UA $(P<0.0001)$, flares $(P<0.0001)$, $P G A(P<0.0001)$, TJC and SJC (both $P<0.0001)$, HAQ-DI $(P=0.02)$, and SF-36 Bodily Pain $(P<0.0001)$. Results for patients without clinically apparent tophi at baseline indicated significant improvements in serum UA $(P<0.0001)$, flares $(P=0.004)$, $P G A(P=0.009)$, TJC $(P=0.01)$, SJC $(P=0.003)$, SF-36 Bodily Pain $(P=0.03)$, and ASHI $(P=0.0001)$.

Conclusions: These results indicate that chronic refractory gout patients may present with or without clinically apparent tophi. Tophaceous patients are distinguished by more tender and swollen joints, greater disability, and greater arthritis severity, but otherwise are similar to nontophaceous patients. Both groups had significant clinical benefit over 6 months of treatment with pegloticase.

References:

[1] Sundy JS, Baraf HS, Yood RA, et al. JAMA. 2011;306:711-720.

[2] Baraf HS, Becker MA, Gutierrez-Urena SR, et al. Arthritis Res Ther. 2013:15:R137.

Disclosure of Interest: N. L. Edwards Consultant for: AstraZeneca, Horizon Pharma, Ironwood Pharmaceuticals, SOBI International, J. Singh: None declared, O. Troum Shareholder of: Theralogix, Grant/research support from: Abbvie, Amgen, CORONA, Novartis, Pfizer, R-Pharm, Consultant for: Abbvie, Amgen, BMS, Pfizer, Roche, Genentech, Speakers bureau: Abbvie, Amgen, BMS, Celgene, Novartis, Pfizer, Roche, Genentech, A. Yeo Consultant for: Horizon Pharma, P. Lipsky Consultant for: AstraZeneca, Celgene, EMD Serono, GSK, Horizon Pharma, Janssen, Medimmune, Pfizer, Roche, Sanofi, UCB

DOI: 10.1136/annrheumdis-2017-eular.4077

\section{THU0449 EVIDENCE BASED DEVELOPMENT OF CRITERIA FOR COMPLETE RESPONSE IN PATIENTS WITH CHRONIC REFRACTORY GOUT}

N. Schlesinger ${ }^{1}$, P. Khanna ${ }^{2}$, A. Yeo ${ }^{3}$, P. Lipsky ${ }^{4} .{ }^{1}$ Department of Medicine, Rutgers Robert Wood Johnson Medical School, New Brunswick, NJ; ${ }^{2}$ Rheumatology, University of Michigan, Ann Arbor, MI; ${ }^{3}$ Horizon Pharma, Lake Forest, IL; ${ }^{4}$ AMPEL BioSolutions, LLC, Charlottesville, VA, United States

Background: Preliminary criteria for remission in gout patients have recently been proposed. These include serum urate, acute flares, tophus, pain and patient global assessment. ${ }^{1}$ These preliminary criteria were based on consensus exercises and have not yet been tested in a large clinical trial database of chronic gout patients. Because of the availability of clinical results from subjects with chronic refractory gout treated with pegloticase ( $8 \mathrm{mg}$ every 2 weeks), a mammalian recombinant uricase conjugated to polyethylene glycol that is approved in the US for treatment of adult patients with chronic gout refractory to oral urate lowering therapy ${ }^{2}$, the utility of these proposed criteria could be assessed.

Objectives: To test the utility of the preliminary criteria to discern a complete response $(\mathrm{CR})$ in subjects with chronic refractory gout treated with pegloticase ( 8 mg every 2 weeks)

Methods: Data from two randomized clinical trials (RCT) evaluating the impact of pegloticase therapy in subjects with chronic refractory gout were examined. ${ }^{2}$ Of this group of subjects, $42 \%$ had persistently lowered serum urate and $58 \%$ did not meet the urate-lowering endpoint of this RCT. Initially, individual patient data was reviewed to establish the frequency with which subjects, who were responders to pegloticase, met the proposed remission criteria. Mixed modeling was then employed on data from these subjects to determine the components of the model that best correlated with time of maximum benefit.

Results: Of 34 pegloticase responders, 25 (73.5\%) met the published criteria ${ }^{1}$ of remission. However, pain assessment was often an outlier; data obtained by visual analogue scale and Medical Outcomes Study Short Form-36 questionnaire often differed. Mixed modeling was, therefore, carried out using the data obtained from the subjects meeting criteria for remission to determine the components that best correlated with time to maximum benefit. Other clinical outcome measures assessed in the clinical trial were also analyzed. Besides serum urate levels in the mixed modeling analysis, the components of response that best correlated with time of maximum benefit included assessment of tophi (analyzed photographically), number of swollen joints, number of tender joints and patient global assessment. Using these criteria, 25 of the responders $(73.5 \%)$ and $29.4 \%$ of the entire pegloticase-treated population met criteria for a CR. The median time to reach a CR was 252 days (range: 126-966 days). Of interest, when a decrease in serum urate was omitted, $6(12.2 \%)$ of the pegloticase nonresponders also met criteria for a CR. Patients receiving placebo did not achieve the composite outcome measure considered as CR.

Conclusions: These results have defined criteria for achieving CR in individuals with chronic refractory gout treated with pegloticase and suggest that most individuals who persistently lowered their serum urate levels while on pegloticase reached criteria for $\mathrm{CR}$ in a median of 8.4 months. This composite $\mathrm{CR}$ definition can serve as an evidence-based target aiding the design and endpoints of future clinical trials.

\section{References:}

[1] deLautour H, Taylor WJ, Adebajo A, et al. Arthritis Care Res. 2016:68:667-672.

[2] Sundy JS, Baraf HSB, Yood RA, et al. JAMA. 2011;306:711-720.

Disclosure of Interest: N. Schlesinger Grant/research support from: As 